\title{
Lernschwierigkeiten und ADHS - eine häufige Kombination?
}

\begin{abstract}
Bei Kindern mit Lernschwierigkeiten werden häufig auch Beeinträchtigungen in der Aufmerksamkeitssteuerung sowie eine vermehrte Hyperaktivität und Impulsivität beobachtet. In einer aktuellen Studie wird der Frage nachgegangen, wie häufig Lernschwierigkeiten mit einer Aufmerksamkeitsdefizit-/ Hyperaktivitätsstörung (ADHS) korreliert sind.
\end{abstract}

\begin{abstract}
Dis ie Autoren untersuchten für ihre Studie 273 Kinder mit Lernschwierigkeiten, 57 Kinder mit unterdurchschnittlicher Intelligenz und 270 Kontrollkinder ohne Lernauffälligkeiten auf das Vorliegen einer ADHS. Die nonverbale Intelligenz und die Schulleistungen wurden über standardisierte Leistungstests erhoben (CFT 1, ELFE 1-6, WRT 2+, DE-Mat 2+), die ADHS-Symptomatik erfolgte über Befragung der Eltern anhand des Fremdbeurteilungsbogen FBB-ADHS.

Die Ergebnisse zeigen, dass 5\% der Kinder der Kontrollgruppe einem ADHS-Subtyp nach DSM-IV entsprachen. Gleiches gilt auch für Kinder, die eine Rechenschwäche aufwiesen. Hingegen erfüllten $20 \%$ der Kinder mit einer Lese-/Rechtschreibschwäche sowie $20 \%$ der Kinder mit unterdurchschnittlicher Intelligenz zusätzlich die Kriteri-
\end{abstract}

en einer ADHS, überwiegend des unaufmerksamen Subtyps.

Die Autoren schließen daraus, dass Kinder mit Lese-/Rechtschreibstörungen und unterdurchschnittlicher Intelligenz ein deutlich erhöhtes Risiko für eine ADHS überwiegend des unaufmerksamen Subtyps haben. Bei einer Rechenschwäche besteht jedoch kein erhöhtes Risiko für eine ADHS

Schuchardt K et al. Die Komorbidität von Lernschwierigkeiten mit ADHS-Symptomen im Grundschulalter. Zeitschrift für Kinder- und Jugendpsychiatrie und Psychotherapie 2015; 43,185-93

\section{Kommentar}

Schon aus früheren Arbeiten ist bekannt, dass Lernstörungen mit einer ADHS assoziiert sein können. Dabei werden je nach Untersuchungsmethode sehr schwankende Werte zwischen 20 und $50 \%$ angegeben.
Dies entspricht auch der Praxis: Nicht selten werden eine Lese-/Rechtschreibstörung oder Rechenschwäche vor der mehr Aufmerksamkeit auf sich ziehenden ADHS übersehen. Aber auch umgekehrt wird bei einer bereits diagnostizierten Lernstörung nicht bemerkt, dass zusätzlich eine ADHS besteht. Bei fehlenden Fortschritten unter einer Lerntherapie sollte immer an diese Möglichkeit gedacht werden.

Methodisch zeigt die vorliegende Arbeit aber mehrere Schwächen: Die Intelligenz anhand eines CFT (Culture Fair Test) zu beurteilen, ist sehr umstritten. Gerade im CFT macht sich ein Aufmerksamkeitsdefizit negativ bemerkbar. So können unterdurchschnittliche Ergebnisse im CFT durchaus auch von einem Aufmerksamkeitsdefizit mit unterdurchschnittlichem Arbeitsgedächtnis und nicht aufgrund von Minderbegabung verursacht sein. Auch die Diagnose ADHS lediglich anhand eines von den Eltern ausgefüllten FBB-ADHS zu stellen, halte ich für sehr fragwürdig. Es entspricht weder den europäischen Leitlinien noch denen der Kinder- und Jugendärzte.

Von daher sagt die Studie lediglich aus, dass eine Leserechtschreibschwäche überdurchschnittlich häufig mit einem auffälligen von den Eltern durchgeführtem Rating im FBB-ADHS assoziiert ist.

Dr. Kirsten Stollhoff

\section{Wie entwickeln sich Vorschulkinder mit ADHS?}

Was ist aus den Kindern mit ADHS geworden, die im Vorschulalter eine medikamentöse Therapie erhielten? Die Autoren der PAT-Studie untersuchten die teilnehmenden Patienten nach 3 und 6 Jahren erneut, um dies herauszufinden.

$\mathrm{D}$ ie Wissenschaftler aus den USA um Benedetto Vitiello stellen jetzt in Form einer systematischen, prospektiven, naturalistischen Studie den Langzeitverlauf der Kinder vor, die 2006 an der „Preschool ADHD Treatment (PAT)"-Studie teilgenommen hatten. 206 (68\%) der ursprünglich 303 Studienteilnehmer konnten 3 Jahre später im Alter von 7,4 Jahren erfasst werden und 197 (59,1\%) Kinder 6 Jahre später im Alter von 10,4 Jahren. Die Daten wurden anhand von Elterninterviews erhoben.
Nach 3 Jahren nahmen $34 \%$ der Kinder mit ADHS keine Medikamente mehr ein, $65 \%$ waren unter Medikation: davon $41,3 \%$ mit einer Monostimulanzientherapie mit Methylphenidat, 9,2\% mit Atomoxetin und 8,3\% nahmen Antipsychotika meist in Kombination mit Stimulanzien ein. 7,2\% erhielten andere Medikamente.

Nach 6 Jahren war der Anteil der Kinder ohne Medikation auf 26,8\% gesunken und der mit Medikation auf $70,9 \%$ gestiegen: $40,2 \%$ erhielten eine Monostimulanzientherapie mit $\mathrm{Me}$ - thylphenidat, 4,5\% entweder nur Atomoxetin oder einer Kombination von Stimulanzien und Atomoxetin, 13,4\% nahmen meist zusätzlich Antipsychotika und $15,9 \%$ andere Medikamente ein. Antipsychotika - meist Risperidon wurden überwiegend Kindern mit zusätzlich disruptiven und pervasiven Verhaltensstörungen verabreicht.

Vitiello B et al. Pharmacotherapy of the Preschool ADHD Treatment Study (PATS) Children Growing Up. J Am Acad Child Adolesc Psychiatry 2015;54:550-6

\section{Kommentar}

Es ist nicht weiter verwunderlich, dass 3 Jahre nach Beginn der medikamentösen Therapie diese nur noch von $65 \%$ der Kinder mit AHDS eingenommen wurde. In der Ursprungsstudie litten viele Kinder unter 